\title{
Efficient generation of stable, heritable gene edits in wheat using CRISPR/Cas9
}

\author{
Rhian M Howells, Melanie Craze, Sarah Bowden and Emma J Wallington * (D)
}

\begin{abstract}
Background: The use of CRISPR/Cas9 systems could prove to be a valuable tool in crop research, providing the ability to fully knockout gene function in complex genomes or to precisely adjust gene function by knockout of individual alleles.

Results: We compare gene editing in hexaploid wheat (Triticum aestivum) with diploid barley (Hordeum vulgare), using a combination of single genome and tri-genome targeting. High efficiency gene editing, $11-17 \%$ for single genome targeted guides and 5\% for tri-genome targeted guides, was achieved in wheat using stable Agrobacterium-mediated transformation. Gene editing in wheat was shown to be predominantly heterozygous, edits were inherited in a Mendelian fashion over multiple generations and no off-target effects were observed. Comparison of editing between the two species demonstrated that more stable, heritable edits were produced in wheat, whilst barley exhibited continued and somatic editing.

Conclusion: Our work shows the potential to obtain stable edited transgene-free wheat lines in 36 weeks through only two generations and that targeted mutagenesis of individual homeologues within the wheat genome is achievable with a modest amount of effort, and without off-target mutations or the need for lengthy crossing strategies.
\end{abstract}

Keywords: Triticum aestivum, Hordeum vulgare, CRISPR\Cas9, Genome editing, Heritability, Knockout

\section{Background}

The ability to examine valuable agronomic traits within crop species has, until recently, been possible only by extensive crossing programmes or the use of genetic modification to insert or silence target genes. The use of RNAi silencing in complex polyploid species mostly results in reduced expression rather than complete knockout, making phenotypic evaluation difficult [1]. Recent developments in the area of gene editing could, therefore, be invaluable as a means of reliably producing true knockouts, particularly in polyploid species.

The use of CRISPR (clustered regularly interspaced short palindromic repeats)/Cas9 systems is increasingly reported in plant species. The system requires two components to be expressed within the same plant cell, the Cas9 adapted from Streptococcus pyogenes and a short guide RNA (gRNA) which targets the Cas9 to the required genomic sequence. Despite

\footnotetext{
* Correspondence: emma.wallington@niab.com

The John Bingham Laboratory, NIAB, Huntingdon Road, Cambridge CB3 OLE, UK
}

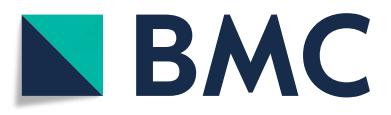

(c) The Author(s). 2018 Open Access This article is distributed under the terms of the Creative Commons Attribution 4.0 International License (http://creativecommons.org/licenses/by/4.0/), which permits unrestricted use, distribution, and

reproduction in any medium, provided you give appropriate credit to the original author(s) and the source, provide a link to the Creative Commons license, and indicate if changes were made. The Creative Commons Public Domain Dedication waiver (http://creativecommons.org/publicdomain/zero/1.0/) applies to the data made available in this article, unless otherwise stated.

the progress made in plants, gene editing remains challenging in transformation-recalcitrant species, with relatively few experiments reported in the major crop species. Within the monocots, editing has been reported in maize, rice and barley [2-4]; however, questions remain from these studies regarding both the editing efficiency and heritability of the induced edits $[5,6]$.

In bread wheat (Triticum aestivum), editing has been reported in protoplast systems $[7,8]$ and, more recently, edited wheat plants have been regenerated after transient expression of Cas9 and guides introduced via particle bombardment [9-11]. However, both the design of the gRNA and the downstream analysis of potential edits are more difficult due to the complex hexaploid nature of the wheat genome. Only limited publications exist on the production of full grown stable edited wheat plants, rather than protoplast systems and all have used a biolistic approach. This method often results in very high copy number complex insertions at multiple loci, as a consequence it can be difficult to remove the Cas9/guide 
cassette in subsequent generations, whilst also segregating the possible bi-allellic edits in a hexaploid genome. Here we present the first example of gene editing in wheat using Agrobacterium-mediated transformation.

To facilitate identification of homeologue specific gene knockouts we have targeted phytoene desaturase (PDS) which is present as a single gene in the wheat genome. PDS is a carotenoid pathway enzyme, which performs the desaturation of phytoene to zeta-carotene [12]. The reduction or loss of function of PDS has been shown to result in a photobleaching phenotype and has been widely used in plants as a visual screen for gene knockout. Virus-induced gene silencing (VIGS; [13]) and gene silencing via RNAi [14] have both been demonstrated using wheat PDS (TaPDS). The conserved nature of PDS between species and the potential to visually phenotype knockout lines make it an ideal candidate gene. In this study we employ it to analyse both the ability to edit genes, and to compare the efficiency and characteristics of the gene editing cassette components in wheat and barley.

The complexity of wheat has meant that genes and regulatory sequences transferred from other, even monocot, species function differently and, therefore, constructs and new technologies can require a greater degree of optimisation. Accordingly, we designed a suite of constructs that enabled testing of different cassette components, including Pol III promoters and gRNA scaffolds in order to progress towards the optimum vector design for gene editing in wheat. Here we demonstrate the production of edited wheat plants using Agrobacterium-mediated transformation. Edits have been produced at a high efficiency without off-target effects and have been shown to be stably inherited through multiple generations to produce lines without the T-DNA bearing the Cas9 and guide cassette sequences.

\section{Results}

Our target gene, TaPDS, is present in wheat as a single copy on each of the three constituent genomes. To exclude any potential for varietal SNPs which might affect gene editing, the first five exons and four introns of all three homoeologues were cloned and sequenced from a US spring wheat variety, Fielder, which is the preferred cultivar for efficient transformation. Comparison of the resulting genomic sequences from Fielder (Additional file 1: Figure S1) showed that the percentage identity of this portion of TaPDS between the three homoeologues was 95-96\%. Sufficient differences were identified within the introns of TaPDS homoeologues to enable design of genome specific primers for PCR amplification (Additional file 2: Table S1), while exonic similarity was sufficient to allow gRNA sequences to be identified for both genome-specific edits and to target all three homoeologues simultaneously with a guide of $100 \%$ match (Additional file 1: Figure S1). The DNA sequence similarity of TaPDS to the orthologous gene in barley (Hordeum vulgare, cv. Golden Promise) was such that, in some cases, the same gRNA could be used for editing in both species. This ability to use the same construct within both to target PDS would not only facilitate analysis across monocot species but also provide a unique ability to compare the efficiency and stability of gene editing between two species.

A total of six constructs (Table 1, Fig. 1) were used in a series of stable Agrobacterium-mediated transformations in wheat, with one of these, pRMH110, also used for Agrobacterium-mediated barley transformation. These constructs enabled analysis of the targeting efficiency within multiple and single genomes, a study of the potential for off-target effects, comparison of editing between wheat and barley and an examination of the efficiency of gene editing per se.

\section{Gene editing targeted to three genomes}

Vector pRMH110 contains a tri-genome targeted guide designed to edit all three wheat PDS homoeologues (Tables 1 and 2, Additional file 1: Figure S1). Thirty-eight transgenic $T_{0}$ wheat lines were generated and edits identified by Sanger sequencing. Two lines were shown to contain heterozygous edits, however, while the guide was tri-genome targeted the resulting edits where only observed within single gene homoeologues. Transgenic lines GE1-2 and GE1-31 were edited in the A and B TaPDS homoeologues, respectively (Table 3). The nature of the edits were confirmed by cloning and Sanger sequencing multiple colonies containing the target TaPDS PCR amplicons. In both cases, the edit was shown to be a single base pair deletion $4 \mathrm{bp}$ from the protospacer adjacent motif site (Table 3, Additional file 3: Table S2). The use of a tri-genome targeting gRNA has, therefore, resulted in an editing efficiency of $5.3 \%$ (Table 4) of single TaPDS homoeologues. This efficiency is comparable to those previously reported in wheat [10].

Table 1 Details of constructs used

\begin{tabular}{lll}
\hline Construct name & Wheat genome targeted & Pol III promoter \\
\hline pRMH110 & A/B/D & TaU6 \\
pRMH120/123 & A & TaU6 \\
pRMH121 & B & OsU3 \\
pRMH125 & D & TaU3 \\
pRMH131 & A/B/D and A only & OsU3 and TaU6 \\
\hline
\end{tabular}




Fig. 1 schematic of binary plasmid T-DNA region transferred to wheat or barley. All constructs conform to this structure with differences as
indicated in Table 1 for the specific Pol III promoters included

\section{Individual genome targeting and off-target edits}

The use of CRISPR gene editing on individual gene homoeologues is dependent on the identification of target sequences with a sufficient level of difference. The level of similarity between wheat homoeologues is often high, resulting in limited options for homoeologue specific guide design. To further analyse this, we produced constructs designed to target the individual genomes of wheat (Table 1); the guides designed are identical to the targeted genome but have differing degrees of similarity to the other two genomes (Table 2). This not only provided a starting point for the evaluation of the degree of similarity possible without increasing the levels of off-target effects, but also provided a means by which the functionality of the Pol III promoter options could be tested in wheat.
Only limited options were available to enable targeting of the B genome homoeologue, and in accordance with the literature, where additional nucleotides have been added to the start of the gRNA without deleterious effect [15-17], an additional adenine residue was added to enable this to be transcribed from the OsU3 promoter. Successful editing was achieved for two of the three guides; vector pRMH125 targeting the D genome homeologue and vectors pRMH120 and pRMH123 targeting the A homeologue. These produced editing efficiencies of $11.4,11.5$ and $16.7 \%$ respectively (Table 4), a higher editing efficiency than that previously published $[7,8]$.

The use of individual genome targeting has also enabled the potential for off-targets to be studied using the other TaPDS homoeologues. The D genome guide has a very high specificity with $11 \mathrm{bp}$ at the $3^{\prime}$ end of the

Table 2 Degree of similarity of individual genome guides to homeologous genes in wheat

\begin{tabular}{|c|c|c|c|c|}
\hline Vector & Genome targeted & Genome & Target sequence & Mismatches (bp) \\
\hline \multirow[t]{5}{*}{$\mathrm{pRMH} 110$} & All genomes & gRNA & TTGTTGCCAAGATTTTCCA & \\
\hline & & A & TTGTTGCCAAGATTTTCCA & $\mathrm{n} / \mathrm{a}$ \\
\hline & & B & TTGTTGCCAAGATTITCCA & $\mathrm{n} / \mathrm{a}$ \\
\hline & & $\mathrm{D}$ & TTGTTGCCAAGATTTTCCA & $\mathrm{n} / \mathrm{a}$ \\
\hline & & Barley & TTGTTGGCAAGATTTTCCA & $\mathrm{n} / \mathrm{a}$ \\
\hline \multirow[t]{4}{*}{ pRMH120 and pRMH123 } & A genome & gRNA & CTGTGTATGAAGTTGTCCGG & \\
\hline & & A & CTGTGTATGAAGTTGTCCGG & $\mathrm{n} / \mathrm{a}$ \\
\hline & & B & CTGCGTATGAAGTTGTCCAG & 2 \\
\hline & & $\mathrm{D}$ & CTGTGTATGAAGTTGCCCAG & 2 \\
\hline \multirow[t]{4}{*}{ pRMH121 } & B genome & gRNA & CTGCGTATGAAGTTGTCCAGC & \\
\hline & & A & CTGTGTATGAAGTTGTCCGGC & 2 \\
\hline & & B & CTGCGTATGAAGTTGTCCAGC & $\mathrm{n} / \mathrm{a}$ \\
\hline & & $\mathrm{D}$ & CTGTGTATGAAGTTGCCCAGC & 2 \\
\hline \multirow[t]{4}{*}{ pRMH125 } & D genome & gRNA & GCCAGGGGAAGTCGAACTAA & \\
\hline & & A & GCCAAGGGA-------------- & 11 \\
\hline & & B & GCCAAGGGA----------_-- & 11 \\
\hline & & $\mathrm{D}$ & GCCAGGGGAAGTCGAACTAA & $\mathrm{n} / \mathrm{a}$ \\
\hline
\end{tabular}

\footnotetext{
'- absence of nucleotide, bold nucleotide substitution
} 
Table 3 Type of edits observed in wheat To plants from different guides

\begin{tabular}{|c|c|c|c|c|}
\hline Genome target & Plant ID & Copy number & Edit type $^{a}$ & Mutation detected $(\mathrm{bp})^{\mathrm{b}}$ \\
\hline \multirow[t]{2}{*}{$\mathrm{A} / \mathrm{B} / \mathrm{D}$} & GE1-2 & $4+$ & Het A & -1 \\
\hline & GE1-31 & $4+$ & Het B & -1 \\
\hline \multirow[t]{6}{*}{ A only } & GE6-4 & $4+$ & Bi-allelic A & $-2 /-16$ \\
\hline & GE6-8 & $4+$ & Bi-allelic A & $+1 /-2$ \\
\hline & GE6-22 & $4+$ & Bi-allelic A & Complex ${ }^{c}$ \\
\hline & GE6-30 & 3 & Het A & -1 \\
\hline & GE6-31 & $4+$ & Het A & +1 \\
\hline & GE6-53 & 4 & Bi-allelic A & $+1 /+1$ \\
\hline \multirow[t]{4}{*}{ D only } & GE7-5 & 3 & Het D & -1 \\
\hline & GE7-10 & $4+$ & Het D & -2 \\
\hline & GE7-21 & $4+$ & Het D & +1 \\
\hline & GE7-28 & $4+$ & Het D & +1 \\
\hline \multirow[t]{5}{*}{ A only } & GE8-1 & $4+$ & Het A & $\mathrm{n} / \mathrm{a}$ \\
\hline & GE8-15 & $4+$ & Het A & -2 \\
\hline & GE8-30 & 2 & Het A & -2 \\
\hline & GE8-31 & 1 & Het A & +1 \\
\hline & GE8-36 & $4+$ & Het A & +1 \\
\hline \multirow[t]{13}{*}{ A/D only Co-transformation } & GE11-13 & $4+$ & Het D & -12 \\
\hline & GE11-23 & $4+$ & Het A & +1 \\
\hline & GE11-24 & $4+$ & Het D & -15 \\
\hline & GE12-1 & 2 & Het A & +2 \\
\hline & GE12-14 & $4+$ & Het D & -3 \\
\hline & GE13-2 & 3 & $\mathrm{D}$ & $\mathrm{n} / \mathrm{a}$ \\
\hline & GE13-8 & $4+$ & Het D & +1 \\
\hline & GE13-28 & $4+$ & Het D & -16 \\
\hline & GE13-36 & $4+$ & Het D & -12 \\
\hline & GE13-38 & $4+$ & Het D & +1 \\
\hline & GE13-42 & 4 & Het A & -1 \\
\hline & GE13-50 & 1 & Bi-allelic D & $-12 /-2$ \\
\hline & GE13-51 & $4+$ & Het D & -7 \\
\hline \multirow[t]{4}{*}{$\mathrm{A} / \mathrm{B} / \mathrm{D}$ and $\mathrm{A}$ double vector } & GE15-1 & 1 & Het A & -2 \\
\hline & GE15-16 & $4+$ & Het A & +1 \\
\hline & GE15-22 & 3 & A & Chimeric \\
\hline & GE15-28 & $4+$ & Het A & +1 \\
\hline
\end{tabular}

${ }^{a}$ Het heterozygous, Bi-allelic two different edits on the same genome copy; letter indicates edited genome,

b $-/+=$ deletion or insertion and number of base pairs involved, $n / a$ data not available

${ }^{c}$ Complex edit which contains both inserts and deletions. For sequences see Additional file 3: Table S2.

guide absent in the sequence of both the $\mathrm{A}$ and $\mathrm{B}$ genome homeologues. While the A genome targeted guide has only 2 nucleotides differing from the B and D homoeologues, sequencing showed that no off-target edits were produced in non-targeted homoeologues.

No edited plants were obtained with the B genome targeted pRMH121 vector, suggesting either the OsU3 guide scaffold does not work as efficiently in wheat in directing the Cas9 to the target sequence, the gRNA design was not optimal or that the additional A nucleotide reduced the efficiency of the editing by weakening the stability of the guide.

Comparing the efficiencies of the successful single genome targeting experiments (Table 4), it is possible to see that the editing is higher than that produced by the tri-genome targeted guide and that the vectors have 
Table 4 Transformation and editing efficiencies

\begin{tabular}{|c|c|c|c|c|c|c|}
\hline Experiment & Construct & Genome target & $\begin{array}{l}\text { No. of inoculated } \\
\text { embryos }\end{array}$ & $\begin{array}{l}\text { No. of transgenic } \\
\text { lines }\end{array}$ & $\begin{array}{l}\text { No. of edited lines (editing } \\
\text { efficiency, \%) }\end{array}$ & $\begin{array}{l}\text { Embryo to edit } \\
\text { efficiency }\end{array}$ \\
\hline GE1 & pRMH110 & $A / B / D$ & 248 & 38 & $2(5.3)$ & 0.8 \\
\hline $\mathrm{GE} 2 / 4$ & pRMH121 & B only & 157 & 28 & $0(0)$ & - \\
\hline GE6 & pRMH120 & A only & 227 & 52 & $6(11.5)$ & 2.6 \\
\hline GE7 & pRMH125 & D only & 213 & 35 & $4(11.4)$ & 1.9 \\
\hline GE8 & pRMH123 & A only & 210 & 30 & $5(16.7)$ & 2.4 \\
\hline $\begin{array}{l}\text { GE11/12/ } \\
13^{\mathrm{b}}\end{array}$ & $\begin{array}{l}\text { PRMH120 / } \\
\text { pRMH125 }\end{array}$ & $A$ and $D$ & 432 & 73 & $13(17.8)$ & 3 \\
\hline GE15/16 & pRMH131 & $\begin{array}{l}\text { A and all three } \\
\text { double }\end{array}$ & 223 & 27 & $4(14.8)$ & 1.8 \\
\hline Hv6 & pRMH110 & N/A & 66 & 40 & $6\left(15^{\mathrm{a}}\right)$ & 9 \\
\hline
\end{tabular}

GE Wheat experiments, $H v$ Barley experiment

${ }^{a}$ High levels of somatic editing were observed making the true edit efficiency unclear

${ }^{b}$ Co-transformation of two different Agrobacterium strains containing different constructs

similar efficiencies regardless of the stringency of the guide to the genome or the promoter used, with the exception of those containing OsU3.

Using our high efficiency wheat transformation method, the single genome targeted guides produce an inoculated embryo to edited plant efficiency of $1.9-3 \%$.

\section{Co-transformation of single genome guides}

Given the higher efficiency achieved with single genome guides compared with tri-genome guides, a possible method by which multiple genome editing could be achieved would be to use two Agrobacterium cultures, each containing a separate construct, in a co-transformation experiment. Constructs pRMH120 (A genome) and pRMH125 (D genome) were co-transformed using a 50:50 ratio; 73 transformed lines were obtained with an overall editing efficiency of $17.8 \%$, the highest seen across all the experiments. Identification of which T-DNA was present within each of the plants showed that the pRMH120 T-DNA (A genome guide) was present in 59\% of plants while the pRMH125 T-DNA (D genome guide) was found in $74 \%$ of plants. Taking the T-DNA presence into account it is possible to determine that not only was the gRNA for the $\mathrm{D}$ genome present in more lines, but also that the efficiency of editing was substantially increased with $26.6 \%$ of plants edited, compared to the $11.4 \%$ achieved when transformed individually (Table 5). In contrast, the efficiency of the A genome specific gRNA was reduced from 11.5 to

Table 5 Percentage of transgenic wheat plants edited in cotransformation experiment compared to when transformed alone

\begin{tabular}{llll}
\hline Genome & Vector & \multicolumn{2}{l}{ Transformation efficiency (\%) } \\
\cline { 3 - 4 } target & & Single constructs & Co-transformation \\
\hline A & pRMH120 & 11.5 & 5.3 \\
D & pRMH125 & 11.4 & 26.6 \\
\hline
\end{tabular}

$5.3 \%$ in the co-transformation experiment (Table 5). In 33\% of lines, both T-DNA copies were present and an equal ratio of editing efficiency was observed for both homoeologues. Noticeably, no lines with both genomes edited were produced in these experiments.

\section{Larger deletion evaluation}

Finally, a construct was designed to evaluate the possibility of creating larger deletions in wheat, which has been demonstrated using CRISPR/Cas9 in both rice and maize $[3,18]$. Construct pRMH131 contained Cas9 plus two guide sequences; the first, the A genome specific guide expressed from the TaU6 promoter, as used in previous experiments, and the second, a new tri-genome guide expressed from the OsU3 promoter. This, we anticipated, would enable us to create standard edits within the $\mathrm{B}$ and $\mathrm{D}$ genome and had the potential for larger deletions in the A genome.

Sequencing the target gene homoeologues from lines produced with pRMH131 showed a $14.8 \%$ efficiency of A genome editing, in agreement with the previous experiments using this guide. However, no edits were identified in any of the genomes at the multi-genome target site, as with the previous experiments using the OsU3 promoter.

\section{Type of edits}

Sequencing the target gene homoeologues in each $\mathrm{T}_{0}$ plant enabled us to further examine the type of edit produced in our system (Table 3). Across all experiments, $50 \%$ of mutations are single base pair insertion or deletion edits, as seen in other species [19] and, overall, $58.8 \%$ of all edits are deletions. It would also appear that guide design may have an impact on the type of edit being created as the A genome guide from pRMH120 and pRMH123 produced $22.2 \%$ of bi-allelic edits whereas the D genome guide from pRMH125 produced just $8.3 \%$. 


\section{Stable inheritance of the gene edits}

The stable inheritance of edits in planta is a much discussed topic, with reports of unpredictable non-Mendelian inheritance in subsequent generations, and the general conclusion is that some edits detected are somatic in nature $[4,5,20]$. Reports indicate that additional editing occurs in subsequent generations and that stable edits are achieved only after the removal of the transgene [6, 18, 21]. Here, we report the first published work, to date, showing stable Mendelian heritability of edits in transformed wheat lines over multiple generations (Table 3).

To determine both the stability of the edit and the ongoing activity of the CRISPR/Cas9, five edited plus seven non-edited lines were progressed to the $\mathrm{T}_{1}$ generation. None of the lines exhibited any additional editing in the next generation, indicating that editing occurred early and that despite the presence of a functional Cas9, additional edits were not produced. This is in contrast to previously published results in wheat [6]. Analysis was carried out on the $\mathrm{T}_{1}$ progeny of the five selected edited plants, four plants heterozygously edited in the $\mathrm{T}_{0}$ (GE1-2, GE1-31, GE7-5 and GE8-30) while the fifth plant (GE13.50) contains biallelic edits. In all cases the observed $\mathrm{T}_{0}$ edits were seen to segregate in a Mendelian fashion with 1:2:1 ratio confirmed using a $X^{2}$ test (Table 6).

The selected lines cover a range of T-DNA copy number (Table 3), GE1-2 and GE1-31 were selected for further generation analysis despite the high 4+ copy due to the presence of the tri-genome targeting guide within these lines. Given the known activity of the Cas9 in the $T_{0}$ generation it was anticipated that these lines might produce additional edits within the non-edited genomes in subsequent generations. However, no additional edits were seen in the $\mathrm{T}_{1}$ generation despite the continued presence of the T-DNA containing the Cas9-gRNA. This is in contrast to observations within rice $[5,12,19]$, wheat $[6]$ and other species $[4,22]$ where new edits were introduced.

Four $\mathrm{T}_{1}$ lines, GE1-2-6, GE1-31-5, GE7.5-1 and GE7-5 - 12, with confirmed homozygous edits were selected and $\mathrm{T}_{2}$ embryos rescued. As anticipated, the expected edit was seen in $100 \%$ of the $\mathrm{T}_{2}$ lines, and again no new edits were identified. Analysis of T-DNA insert was carried out using both an nptII copy number assay and a Cas9 absence/presence PCR and the results show the expected presence of both full length and partial T-DNA inserts. However, prudent selection of lines at both the $T_{0}$ and $T_{1}$ generation can enable rapid progression to homozygous edited, Cas9 free plants (see Additional file 4: Table S3).

GE7-5-1 was selected in the $T_{1}$ generation using nptII copy number assay with zero copies detected. A single partial T-DNA insertion bearing Cas9 segregated in this line with the expected 1:2:1 ratio, resulting in 25\% Cas9/T-DNA free edited $\mathrm{T}_{2}$ lines identified (Additional file 4: Table S3). GE7-5-12 was identified as 4 copy, and thus, the segregation of the T-DNA would only be expected to produce a limited number of null segregants, however we analysed 23 lines and successfully identified a single null segregating plant (Additional file 4: Table S3). The remaining two lines GE1-2-6 and GE1-31-5 both showed 4+ copies of $n p t I I$ and in the limited seed produced from a single ears, with 16 plants and 24 plants germinated respectively, it was not possible to identify Cas9 free lines. $\mathrm{T}_{0}$ lines with lower nptII copy number such as GE8-30 which contains two T-DNA copies plus a partial T-DNA, can be rapidly progressed through the $\mathrm{T}_{1}$ generation in order to select for the Cas 9 free plants in the $T_{2}$ generation (Additional file 4: Table S3). Even plants indicating a higher nptII copy number, such as GE13-50, are able to produce lower copy number lines within the $T_{1}$ generation, if the edit is considered to be of greater importance (Additional file 4: Table S3).

Given the lack of additional edits observed in subsequent generations it was postulated that a callus stage might be a requirement for the activation of the CRISPR/Cas9 system and the production of edits. To test this hypothesis, $\mathrm{T}_{2}$ immature embryos from a homozygous edited GE1 line were used to generate 251 plantlets via callus induction and subsequent regeneration. Line GE1-31-5 was homozygous for a B genome edit created using a tri-genome targeted guide, and also

Table 6 Segregation of edit in $T_{1}$ plants. Number of plants of each genotype is indicated

\begin{tabular}{lllll}
\hline$T_{0}$ line & Homozygous edit & Heterozygous edit & WT & $X^{2} P$ value \\
\hline GE1-2 & 8 & 8 & 3 & 0.212 \\
GE1-31 & 6 & 14 & 8 & 0.867 \\
GE7-5 & 6 & 14 & 9 & 0.721 \\
GE8-30 & 11 & 27 & 5 & 0.106 \\
To line & Homozygous allele 1 & Bi-allelic & Homozygous allele 2 & $X^{2} P$ value \\
GE13-50 & 7 & 13 & 3 & 0.410 \\
\hline
\end{tabular}


retained a 4+ nptII copy number for the Cas9 T-DNA. Given the presence of a tri-genome targeting guide, additional edits within non-edited genomes might be anticipated if the system is reactivated via a callus induction stage. Our data indicates that this is not the case as while the B genome edit was fully retained, no additional genomic edits were observed.

\section{Comparison of gene editing in barley}

The tri-genome guide contained within pRMH110 is also able to target the barley HvPDS gene, with a $100 \%$ identity for the guide. Forty stable independent transformed barley lines where examined, of which six demonstrated editing. This gives an efficiency of editing for the single $H v P D S$ gene in diploid barley of $15 \%$, compared to $5 \%$ efficiency for the same tri-genome targeting guide in hexaploid wheat. The cloning and sequencing of the potentially edited barley lines showed larger deletions of up to $350 \mathrm{bp}$, and also demonstrated the presence of somatic editing. In contrast to wheat, cloning and sequencing the barley $\mathrm{T}_{0}$ amplicons showed multiple genotypes to be present within a single plant in 5 of the 6 lines, with up to 4 different genotypes within a single leaf sample. This is borne out in the phenotype exhibited by the plants, which showed chimeric photobleaching (Fig. 2). A closer examination of the chimeric

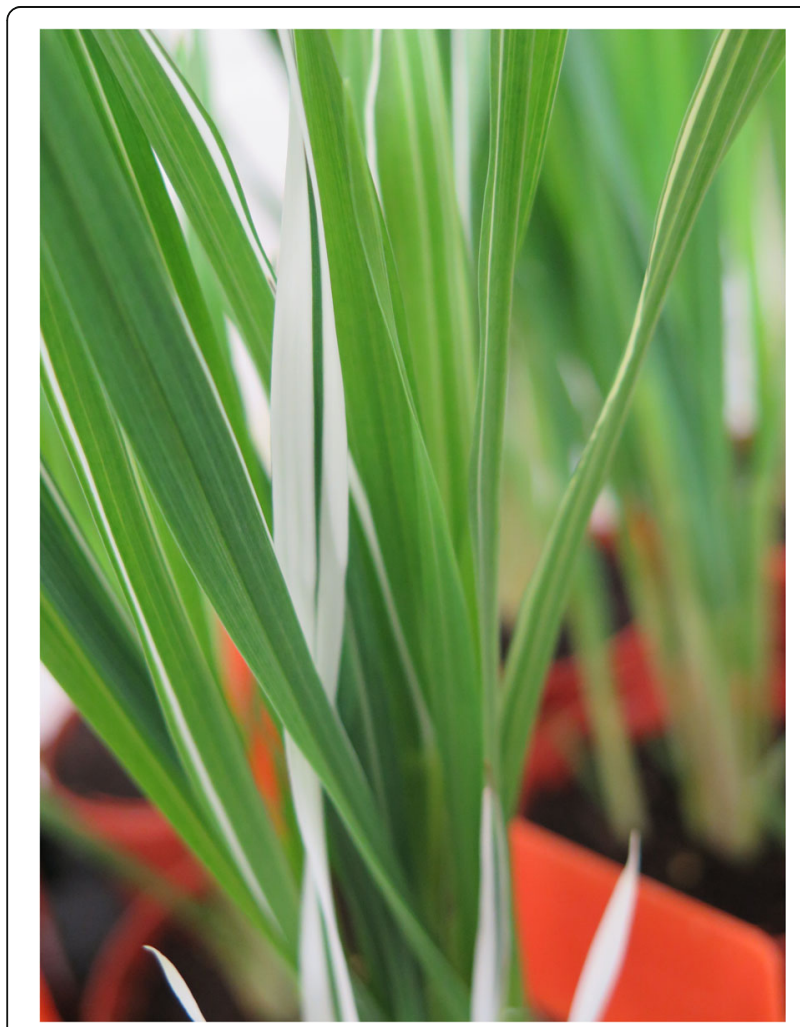

Fig. 2 Transgenic barley plant exhibiting chimaeric photobleaching phenotype regions, using DNA from striped, white and completely green leaf areas, showed that these corresponded to regions where heterozygous and homozygous edits or WT sequences were detected. Re-sampling of plants 44 days later produced a different series of edits suggesting that the edit obtained is specific to the section of leaf rather than true plant genotype (data not shown). Previous published work in barley also suggests that edits could be somatic, which is in line with our results [4]. This is in contrast to our results in wheat, where chimaerism was observed in only one of the 34 edited lines (GE1522) which was detected by sequencing the homeologue target region, and identifying four sequence variants including the WT (Table 3).

\section{Discussion}

The results presented here show that our Agrobacterium-mediated transformation system in wheat is able to produce an editing efficiency equivalent to the previously published efficiencies using other transformation methods [7, 8]. Indeed, this efficiency is within the range commonly reported for standard wheat transformation per se [23]. This demonstrates that targeted mutagenesis of individual homeologues within the wheat genome is achievable with a modest amount of effort, and without associated off-target mutations. While the editing efficiencies observed in wheat were within the ranges anticipated, no edits were obtained using the OsU3 promoter. The increased ability of the gRNAs expressed from the TaU3 promoter to introduce edits compared with OsU3 has previously been noted in maize protoplasts [16] and the lack of edits using OsU3 with either of the two guides, single or tri-genome targeted, would suggest that it is not acting efficiently enough in our wheat experiments to produce edits.

Across all the experiments carried out, edits were produced in each of the three TaPDS homoeologues such that the resultant protein would be truncated, however, no phenotype was observed indicating that the other two genomes can compensate for the loss of a single genome copy. Our targeting of the individual wheat genomes has enabled a closer examination of the potential for generation of off-target edits and our results indicate that even with limited nucleotide differences between the genome sequences, no off-target editing occurred within the non-targeted homeologues. This result was unexpected given previous reports found that off-target effects were seen between the homeologues of wheat genes [10]. Our results indicate that high levels of similarity can be used within this system without resulting in off-target edits, however, a more detailed examination of multiple guides would be required to determine whether 
the position of mismatches within the guide results in increased off-target editing.

The use of co-transformation for editing has demonstrated that while the levels of editing efficiency observed might not produce multiple homeologue knockouts in a single plant, it would provide an excellent means by which different homeologues or genes could be knocked out in wheat via a single transformation experiment. This would not only reduce the time required for multiple plant transformation experiments but also reduce the total number of $\mathrm{T}_{0}$ plants required, and thus, the overall amount of molecular analysis and, therefore, greatly reduce costs.

A single construct containing both guides, rather than using co-transformation with separate guides, would ensure that the editing machinery is being introduced into the same cell and increase the likelihood of a line edited in both genome copies being produced. Conversely, the lower ratio of Cas9 protein per gRNA compared with the single vector system could potentially reduce efficiency. Cas 9 expression could be elevated with the use of a stronger promoter, such as maize ubiquitin, however whether this may also have deleterious effects such as ongoing editing, or higher levels of somatic editing, remains to be determined.

In our wheat experiments, the predominant type of edit introduced was a single nucleotide insertion or deletion as observed in other species [19], however, the percentage of bi-allelic edits was lower than that observed in rice $[5,20]$. This may suggest that a more stringent DNA break repair system is present in wheat. Inter-species differences have also been demonstrated by our observation that the same construct produces different patterns of editing between the two closely related species, wheat and barley. It would appear that in wheat the editing occurs at an early stage and is stable, whereas in barley editing continues to occur (including at somatic level) and is less stable. These observations would indicate, therefore, that for optimum results, both construct components and subsequent analysis should be tailored to the individual species.

Our experiments on inheritance of edits across multiple generations demonstrates the necessity for the early establishment of T-DNA copy number for lines, and that careful selection of lines not only for edit, but also T-DNA copy number, is required to rapidly progress to the successful identification of edited T-DNA free lines. Using embryo rescue it was therefore possible to achieve stable edited Cas9 free $T_{2}$ wheat plants from the initiation of plant transformation in only 36 weeks. This rapid progression to stable edited transgene free $T_{2}$ lines is only made possible as a result of the Mendelian inheritance of the edit, even in the presence of the Cas9 in the $\mathrm{T}_{1}$ generation. Our results appear to contrast with previously published work in monocots $[4,11]$ where edits are of a more complex bi-allelic nature, including chimeric, and $T_{1}$ progeny do not follow the expected Mendelian segregation ratios in the presence of Cas9 or where additional editing is observed [6]. It is unclear at present the reason for this contrasting result but we would postulate that the vector design is the most likely source of this difference.

\section{Conclusions}

Our work shows that it is possible to obtain efficient gene editing in wheat using Agrobacterium-mediated stable transformation of CRISPR/Cas9 constructs; our embryo to edit efficiency is broadly equivalent to the efficiency of wheat transformation alone as reported by other researchers. We demonstrate that the same construct can produce different patterns of editing in two different monocot crops, showing the importance of tailoring both the construct design and the downstream analysis to the individual plant species. Within our system, edits occur early in the wheat transformation process and the edits are stable within the $\mathrm{T}_{0}$ plant and subsequent generations. In contrast, edits continue to occur in barley, and are often somatic in nature. Our results indicate that off-target editing has not occurred in wheat in this study, and that edits are heritable in a Mendelian fashion in presence of Cas9 from the first generation. Whether this is a consequence of the differing ploidy levels in the two species remains unclear. This work demonstrates that it is possible to edit hexaploid wheat and obtain T-DNA/Cas9 free edited plants in a relatively short space of time, without the need to screen large numbers of plants. We demonstrate that co-transformation could be used as an effective means of obtaining multiple edits through a single transformation experiment without loss of efficiency. While other published work has reported the requirement for rapid removal of Cas9 and complex bi-allelic and chimeric editing patterns with continuing editing, our system demonstrates an efficient strategy by which multiple "easy to breed" edits can be obtained from a single transformation experiment. The lack of additional editing in subsequent generations and Mendelian segregation observed, even in the continued presence of Cas9, provides the ability to rapidly progress those lines through multiple generations to obtain T-DNA clean, stable homozygous edited lines for phenotypic evaluation.

\section{Methods}

\section{Design of vectors}

The Cas9 gene sequence [7], including the nuclear localisation signals, was codon optimised for wheat and synthesised (Genscript, Piscataway, NJ, USA), and 
recombined into vector pSc4ActR1R2 [24] using a Gateway Clonase II kit (Thermo Fisher Scientific Inc.). In the resulting vector, pRMH088, the Cas9 gene is expressed from the rice Actin promoter [25] and transcripts terminated by the nopaline synthase terminator (tNOS) from Agrobacterium tumefaciens. The vector which also contains the nptII (neomycin phosphotransferase II) gene expressed from the subterranean clover stunt virus 4 promoter [26] with the Arabidopsis thaliana FAD2 intron [27] and transcripts were terminated by tNOS.

Basic gRNA vectors were designed containing one of three Pol III polymerase promoter and terminators, TaU3 [18], TaU6 from wheat or OsU3 [7] from rice and synthesised in the pUC57 backbone (Genscript, Piscataway, NJ, USA). PDS target guide sequences were inserted into the vectors using annealed primer pairs ligated into unique restriction sites. Each gRNA cassette was then cloned into pRMH088 using a second set of unique restriction sites.

\section{Gene identification}

The wheat phytoene desaturase (TaPDS) gene was identified using the rice PDS gene (GenBank accession AF049356) as a query for a BLAST [28] analysis of the wheat genome reference sequence, IWGSCv1 wheat genome [29]. Gene predictions for all three homoeologues were produced by alignment of each genomic sequence to identified wheat expressed sequence tag (EST) sequences using est2genome [30]. The first five exons and four introns of TaPDS were amplified from the US spring variety Fielder (USDA, ARS) using TaPDS genomic sequence primers (Additional file 1: Figure S1 and Additional file 2: Table S1) and Phusion Hotstart II polymerase (Thermo Fisher Scientific Inc.). Amplicons were cloned into the pGEM-T Easy vector system (Promega), a number of colonies were sequenced and the varietal sequence for the three homoeologues confirmed.

Primers were designed to genome specific SNPs identified from TaPDS sequences (Additional file 2: Table S1) and PCR amplicons tested for genome-specificity using the Chinese Spring nulli-tetrasomic lines [31]. PCR conditions with primers at a final concentration of $1 \mathrm{mM}, 0.4 \mathrm{U}$ FastStart Taq DNA polymerase (Sigma-Aldrich) and using the provided standard $10 \times$ reaction buffer with $\mathrm{MgCl}_{2}$ to a final concentration of $2 \mathrm{mM}$ and dNTPs to a final concentration of $1 \mathrm{mM}$. PCR conditions were all as follows: $\left(95^{\circ}\right.$ C 4 mins $\left[95{ }^{\circ} \mathrm{C} 30 \mathrm{~s}, 64{ }^{\circ} \mathrm{C} 30 \mathrm{~s}, 71{ }^{\circ} \mathrm{C} 1 \mathrm{~min}\right] \times 40,72{ }^{\circ} \mathrm{C}$ 10mins).

Barley $H v P D S$ genomic sequence was identified using the TaPDS homoeologues in a BLAST [28] query of the barley genome assembly $082214 \mathrm{v} 1$, in the variety Morex [32], and aligned with the wheat sequences using AlignX (Thermo Fisher Scientific Inc.). The identified region of similarity was amplified from variety Golden Promise and examined in transgenics with the primers listed in Additional file 2: Table S1 using the PCR conditions above with annealing temperature of $54{ }^{\circ} \mathrm{C}$.

\section{Design of gRNA}

Target sequences were selected within the first two exons of the gene using the Emboss tool DREG [30], with the sequence specification of $\mathrm{G}(\mathrm{N})_{21} \mathrm{GG}$ or $\mathrm{A}(\mathrm{N})_{21} \mathrm{GG}$. Gene specificity was confirmed using a BLAST of the target sequence to the IWGSCv1 wheat genome. Genome specificity and promoter type were used to select the gRNA sequence for each construct.

\section{Plant growth, tissue culture and transformation}

Completed constructs were verified by restriction digest and sequencing before being electro-transformed into Agrobacterium tumefaciens strain AGL1 [33]. Plasmids were isolated from Agrobacterium colonies selected on media containing kanamycin $(50 \mu \mathrm{g} / \mathrm{ml})$ and rifampicin $(50 \mu \mathrm{g} / \mathrm{ml})$ then verified by restriction digest prior to use in wheat or barley transformation experiments [34].

Fielder wheat stock plants (USDA, ARS) were grown in M2 compost plus $5 \mathrm{~g} / \mathrm{l}$ slow release fertilizer (Osmacote Exact 15:9:9) in a controlled environment chamber (Conviron) at $20{ }^{\circ} \mathrm{C}$ day $/ 15{ }^{\circ} \mathrm{C}$ night with a $16 \mathrm{~h}$ photo-

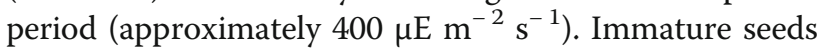
were harvested for transformation experiments at 1420 days post-anthesis (dpa). Wheat embryos were isolated and then co-cultivated with Agrobacterium tumefaciens for 2 days in the dark [35]. The embryonic axis removal and subsequent tissue culture steps were performed as described by Risacher et al. [36]. Individual plantlets were transferred to Jiffy-7 pellets (LBS Horticulture) and hardened off, then potted up into $9 \mathrm{~cm}$ plant pots and grown on to flowering in controlled environment chambers, as above.

Embryo rescue was performed by isolating embryos (12-18 dpa) aseptically, followed by culture, embryo axis uppermost, on MRMZ2 medium (MRM medium [36] supplemented with $2 \mathrm{mg} / \mathrm{l}$ zeatin in place of kinetin). After approximately 5-7 days on MRMZ2, germinated shoots were transferred to 40-well tray inserts containing Levingtons M2 compost with added Osmocote exact slow release fertilizer (LBS horticulture), and grown on in plant growth chambers, as above. For callus induction experiments, immature embryos (14-20 dpa) were isolated aseptically and placed, scutellum uppermost, onto W425G medium [36]. After 3 days, axes were excised and the scutella transferred to fresh W425G. Following a further transfer to W425G, with a total of 6 weeks on selective callus induction medium, calli were transferred to MRMZ2-25G medium for regeneration, followed by transfer of shoots into Beatson jars containing MS20 
medium. After 4 weeks, plantlets were sampled for analysis.

Agrobacterium tumefaciens-mediated transformation of barley variety Golden Promise (JIC, UK) was carried out, using immature embryos, as per published protocols [37].

\section{Plant DNA extraction}

Chinese Spring nulli-tetrasomic lines [31] were grown under poly-tunnel conditions, leaves sampled from individual lines, and genomic DNA extracted using a modified Tanksley extraction method [38].

DNA from transgenic wheat and barley lines was extracted using crude DNA extraction buffer $(200 \mathrm{mM}$ Tris pH 7.5, $250 \mathrm{mM} \mathrm{NaCl}, 25 \mathrm{mM}$ EDTA, 0.5\% SDS), incubated at $65{ }^{\circ} \mathrm{C}$ for $1 \mathrm{~h}$ then centrifuged at $6000 \mathrm{~g}$ for $10 \mathrm{~min}$. The DNA was precipitated by addition of $400 \mu \mathrm{l}$ isopropanol to the supernatant followed by centrifugation, as previously. DNA pellets were resuspended in $100 \mu \mathrm{l}$ TE, incubated at $65^{\circ} \mathrm{C}$ for $5 \mathrm{~min}$ and centrifuged at $6000 \mathrm{~g}$ for $5 \mathrm{~min}$. DNA was diluted 1:3 in sterile $\mathrm{H}_{2} \mathrm{O}$ prior to use in all assays.

\section{Plant analysis}

T-DNA copy number was determined using a TaqMan relative quantification $(\Delta \Delta \mathrm{CT})$ assay comparing the relative values of an nptII amplicon to an amplicon of the single copy wheat gene $G a M y b$ within a multiplexed reaction and normalised to a single copy control [39]. All reactions are carried out using two replicates per plant line. Primers and probes (Additional file 2: Table S1) were used at a final concentration of $10 \mu \mathrm{M}$ in $10 \mu \mathrm{l}$ reactions with ABsolute Blue qPCR ROX mix (Thermo Fisher Scientific Inc.) using the standard run conditions for the ABI 7900 HT (Thermo Fisher Scientific Inc.).

Cas9 presence/absence PCR was carried out using the standard FastStart Taq DNA mix and conditions (as above) with an annealing temperature of $57{ }^{\circ} \mathrm{C}$ and extension time of $1 \mathrm{~min} 30 \mathrm{~s}$.

For determination of edits, genome specific PCRs for all three homoeologous genes were conducted on each transgenic plant. Amplicons were purified using an Exo-SAP reaction (Thermo Fisher Scientific Inc.) and Sanger sequencing carried out using ABI Big Dye Mix v3.1 (Thermo Fisher Scientific Inc.) with the provided protocol, and run on an ABI 3730 instrument (Thermo Fisher Scientific Inc.).

Where the plant line showed an edit based on an initial sequence chromatogram, the amplicon was cloned into pGEM-T Easy (Promega) and 5-20 colonies for each amplicon sequenced as previously. Traces were examined using a combination of Contig Express and AlignX (Thermo Fisher Scientific Inc.).

\section{Additional files}

Additional file 1: Figure S1. Sequence alignment of TaPDS from wheat variety Fielder and HvPDS from barley variety Golden Promise with guide sites indicated. (DOCX $149 \mathrm{~kb}$ )

Additional file 2: Table S1. Sequences of primers used. (XLSX $10 \mathrm{~kb}$ ) Additional file 3: Table S2. Edits identified in To plants. (DOCX 24 kb) Additional file 4: Table S3. Segregation of Cas9 and edits within the $T_{1}$ and $T_{2}$ plants. (XLSX $\left.19 \mathrm{~kb}\right)$

\section{Abbreviations}

CRISPR: Clustered regularly interspaced short palindromic repeats; EST: Expressed sequence tag; gRNA: Guide RNA; nptll: Neomycin phosphotransferase; PDS: Phytoene desaturase; Pol III: Polymerase III; RNAi: RNA interference; SNPs: Single nucleotide polymorphisms;

tNOS: Nopaline synthase terminator; VIGS: Virus induced gene silencing

\section{Acknowledgements}

The authors would like to thank Ruth Bates for additional tissue culture support and Andy Greenland for his encouragement and advice.

\section{Funding}

The authors were funded by the UK Biotechnology and Biological Sciences Research Council (BBSRC) grant BB/J019356/1. The funders had no role in the experimental design, data collection and analysis or preparation of the manuscript.

\section{Availability of data and materials}

The datasets and materials generated and analysed during the current study are available under MTA from the corresponding author on reasonable request.

\section{Authors' contributions}

$\mathrm{RH}$ and EW designed the experiments and constructs. MC and SB generated transgenic lines and performed all tissue culture steps. $\mathrm{RH}$ generated the constructs and performed the molecular characterisation of plants. The manuscript was written by $\mathrm{RH}$ and EW. All authors read and approved the final manuscript.

Ethics approval and consent to participate

Not applicable

\section{Consent for publication}

Not applicable

\section{Competing interests}

The authors declare that they have no competing interests.

\section{Publisher's Note}

Springer Nature remains neutral with regard to jurisdictional claims in published maps and institutional affiliations.

Received: 19 June 2018 Accepted: 20 September 2018

Published online: 03 October 2018

\section{References}

1. Fu D, Uauy C, Blechl A, Dubcovsky J. RNAi interference for wheat functional gene analysis. Transgenic Res. 2007;16:689-701.

2. Svitashev S, Young J, Schwartz C, Gao H, Falco SC, Cigan AM. Targeted mutagenesis, precise gene editing and site-specific gene insertion in maize using Cas9 and guide RNA. Plant Physiol. 2015;169(2):931-45.

3. Zhou H, Liu B, Weeks DP, Spalding MH, Yang B. Large chromosomal deletions and heritable small genetic changes induced by CRISPR/Cas9 in rice. Nucleic Acids Res. 2014;42:10903-14.

4. Lawrenson T, Shorinola O, Stacey N, Li C, Østergaard L, Patron N, Uauy C, Harwood W. Induction of targeted, heritable mutations in barley and Brassica oleracea using RNA-guided Cas9 nuclease. Genome Biol. 2015; 16(1):258. 
5. Ishizaki T. CRISPR/Cas9 in rice can induce new mutations in later generations, leading to chimerism and unpredicted segregation of the targeted mutation. Mol Breed. 2016;36:165.

6. Wang W, Pan Q, He F, Akhunova A, Chao S, Trick H, Akhunov E. Transgenerational CRISPR-Cas9 activity facilitates multiplex gene editing in allopolyploid wheat. CRISPR J. 2018;1(1):65-74.

7. Shan Q, Wang Y, Li J, Zhang Y, Chen K, Liang Z, et al. Targeted genome modification of crop plants using a CRISPR-Cas system. Nat Biotechnol. 2013;31:686-8

8. Kim D, Alptekin B, Budal H. CRISPR/Cas9 genome editing in wheat. Funct Integr Genom. 2018;18:31-41.

9. Wang Y, Cheng X, Shan Q, Zhang Y, Liu J, Gao C, Qiu JL. Simultaneous editing of three homoeoalleles in hexaploid bread wheat confers heritable resistance to powdery mildew. Nat Biotechnol. 2014;32(9):947.

10. Zhang $Y$, et al. Efficient and transgene-free genome editing in wheat through transient expression of CRISPR/Cas9 DNA or RNA. Nat Commun. 2016;7:12617.

11. Zhang Y, Bai Y, Wu G, Zou S, Chen Y, Gao C, Tang D. Simultaneous modification of three homoeologs of TaEDR1 by genome editing enhances powdery mildew resistance in wheat. Plant J. 2017;91(4).

12. Bartley GE, Scolnik PA. Plant carotenoids: pigments for photoprotection, visual attraction, and human health. Plant Cell. 1995;7:1027-38.

13. Scofield SR, Huang L, Brandt AS, Gill BS. Development of a virus-induced gene-silencing system for hexaploid wheat and its use in functional analysis of the Lr21-mediated leaf rust resistance pathway. Plant Physiol. 2005;138: 2165-73.

14. Travella S, Klimm TE, Keller B. RNA interference-based gene silencing as an efficient tool for functional genomics in hexaploid bread wheat. Plant Physiol. 2006;142:6-20.

15. Ran FA, Hsu PD, Wright J, Agarwala V, Scott DA, Zhang F. Genome engineering using the CRISPR-Cas9 system. Nat Protoc. 2013;8(11):2281.

16. Shan Q, Wang Y, Gao C. Genome editing in rice and wheat using the CRISPR/Cas system. Nat Protoc. 2014;9:2395.

17. Xu R, Li H, Qin R, Wang L, Li L, Wei P, Yang J. Gene targeting using the agrobacterium tumefaciens-mediated CRISPR-Cas system in rice. Rice. 2014;7(1):5.

18. Xing $\mathrm{H}$-L, et al. A CRIPSR/Cas9 toolkit for multiplex genome editing in plants. BMC Plant Biol. 2014;14:327.

19. Ma X, Zhang Q, Zhu Q, Liu W, Chen Y, Qiu R, Wang B, Yang Z, Li H, Lin Y, Xie $Y$. A robust CRISPR/Cas9 system for convenient, high-efficiency multiplex genome editing in monocot and dicot plants. Mol Plant. 2015:8(8):1274-84.

20. Xu RF, Li H, Qin RY, Li J, Qiu CH, Yang YC, Ma H, Li L, Wei PC, Yang JB. Generation of inheritable and "transgene clean" targeted genomemodified rice in later generations using the CRISPR/Cas9 system. Sci Rep. 2015;5:11491.

21. Gao X, Chen J, Dai X, Zhang D, Zhao Y. An effective strategy for reliably isolating heritable and Cas9-free Arabidopsis mutants generated by CRISPR/Cas9-mediated genome editing. Plant Physiol. 2016;171:1794-800.

22. Jacobs TB, Zhang N, Patel D, Martin GB. Generation of a collection of mutant tomato lines using pooled CRISPR libraries. Plant Physiol. 2017;174: 2023-37.

23. Li J, Ye X, An B, Du L, Xu H. Genetic transformation of wheat: current status and future prospects. Plant Biotechnol Rep. 2012;6:183-93.

24. Perochon A, Jianguang J, Kahla A, Arunachalam C, Scofield SR, Bowden S, Wallington E, Doohan FM. TaFROG encodes a Pooideae orphan protein that interacts with SnRK1 and enhances resistance to the mycotoxigenic fungus Fusarium graminearum. Plant Physiol. 2015;169(4): 2895-906.

25. McElroy D, Zhang W, Cao J, Wu R. Isolation of an efficient actin promoter in rice transformation. Plant Cell. 2008;2:163-71.

26. Schünmann PH, Llewellyn DJ, Surin B, Boevink $P$, De Feyter RC, Waterhouse PM. A suite of novel promoters and terminators for plant biotechnology. Funct Plant Biol. 2003;30(4):443-52.

27. Okuley J, Lightner J, Feldmann K, Yadav N, Lark E. Arabidopsis FAD2 gene encodes the enzyme that is essential for polyunsaturated lipid synthesis. Plant Cell. 1994;6(1):147-58

28. Altschul SF, Gish W, Miller W, Myers EW, Lipman DJ. Basic local alignment search tool. J Mol Biol. 1990:215:403-10.

29. IWGSC. A chromosome-based draft sequence of the hexaploid bread wheat (Triticum aestivum) genome. Science. 2014;345:6194.
30. Rice P, Longden I, Bleasby A. EMBOSS: the European molecular biology open software suite. Trends Genet. 2000;16:276-7.

31. Sears ER. The aneuploids of common wheat. University of Missouri Agricultural Experiment Station Research Bulletin. 1954;572:1-58.

32. The International Barley Genome Sequencing Consortium. A physical, genetic and functional sequence assembly of the barley genome. Nature. 2012;491:711-6.

33. Lazo GR, Stein PA, Ludwig RA. A DNA transformation-competent Arabidopsis genomic library in agrobacterium. Biotechnology. 1991;9: 963-7.

34. Bates R, Craze M, Wallington EJ. Agrobacterium-mediated transformation of Brassica napus. Curr Protoc Plant Biol. 2017;2:287-98.

35. Ishida Y, Tsunashima M, Hiei Y, Komari T. Wheat (Triticum aestivum L.) transformation using immature embryos. In Agrobacterium Protocols. New York: Springer; 2015. p. 189-198.

36. Risacher T, Craze M, Bowden S, Paul W, Barsby T. Highly efficient Agrobacterium-mediated transformation of wheat via in planta inoculation. In: Jones HW, Shewry PR, editors. Methods in molecular biology, transgenic wheat, barley and oats, vol. 478: Humana Press; 2009. p. 115-24.

37. Harwood, A. A protocol for high-throughput agrobacterium-mediated barley transformation. In: Henry, RJ, Furtado A, editors Cereal Genomics, vol. 1099. Humana Press; 2014. p.251-260.

38. Fulton TM, Chunwongse J, Tanksley SD. Microprep protocol for extraction of DNA from tomato and other herbaceous plants. Plant Mol Biol Rep. 1995:13:207-9.

39. Milner MJ, Howells RM, Craze M, Bowden S, Graham N, Wallington EJ. A PSTOL-like gene, TaPSTOL, controls a number of agronomically important traits in wheat. BMC Plant Biol. 2018;18(1):115

\section{Ready to submit your research? Choose BMC and benefit from:}

- fast, convenient online submission

- thorough peer review by experienced researchers in your field

- rapid publication on acceptance

- support for research data, including large and complex data types

- gold Open Access which fosters wider collaboration and increased citations

- maximum visibility for your research: over $100 \mathrm{M}$ website views per year

At BMC, research is always in progress.

Learn more biomedcentral.com/submissions 